\title{
Tracking for Nonlinear Underactuated Surface Vessels with Generalized Forces
}

\author{
Gregory J. Toussaint \\ Tamer Başar \\ Coordinated Science Laboratory \\ University of Illinois at Urbana-Champaign \\ 1308 West Main Street \\ Urbana, IL 61801
}

Francesco Bullo

\begin{abstract}
This paper describes how to use backstepping to develop control laws to perform trajectory tracking for a nonlinear, underactuated surface vessel. The research extends earlier backstepping designs for underactuated vessels by explaining how to select outputs when generalized forces act on the vessel. The resulting control law can correct orientation errors to track linear trajectories and can track arcs of circles with a fixed offset. The paper provides detailed derivations along with simulation results to illustrate the approach.
\end{abstract}

\section{Introduction}

Integrator backstepping is commonly used to design controllers for nonlinear systems, but using the technique for underactuated nonlinear systems presents some unique challenges. Our objective is to develop a controller that causes a nonlinear underactuated vehicle to track a given reference trajectory. We will use integrator backstepping as the primary design tool and show how to adapt the technique for general forces acting on an underactuated system. The results are based largely on recent contributions by Godhavn [1] and we explain how to extend his approach.

An underactuated system is defined to be one where the dimension of the space spanned by the control vector is less than the dimension of the configuration space [2]. Simply stated, this refers to a mechanical system that has fewer control inputs than degrees of freedom. An equivalent characterization of an underactuated system is that it has nonintegrable acceleration relations or dynamics [9].

The example underactuated vehicle we will focus on is a ship moving on a planar surface that has two forces applied at the rear of the vessel. The ship is underactuated because there are only two acceleration inputs and there are three degrees of freedom on the planar surface, which consist of the two position coordinates $(x, y)$ and the orientation $(\theta)$. Other examples of underactuated vehicles include spacecraft, cer- tain aircraft, hovercraft and missiles [3, 6, 8]. This problem is nonminimum phase if we select the $x$ and $y$ positions as outputs to track. The nonminimum phase property appears because the two forces combine to produce lateral acceleration in addition to forward acceleration and torque.

In addition to being underactuated, the vehicles studied in this effort will have nonlinear equations of motion. Nonlinear systems present their own set of design challenges if we want to plan a set of motions and control the vehicle to follow a specific trajectory. The standard tools used to control nonlinear systems, including feedback linearization and integrator backstepping, do not provide complete solutions for the underactuated systems we will consider. We will show how to modify the standard backstepping procedure to handle an underactuated vehicle with generalized forces under certain conditions.

¿From a practical perspective, there are at least two advantages to designing controllers for underactuated systems. First, a fully actuated system requires more control inputs than an underactuated system, which means there will have to be more devices to generate the necessary forces. The additional controlling devices add to the cost and weight of the system. Finding a way to control an underactuated version of the system would eliminate some of the controlling devices and could improve the overall performance or reduce the cost. The second practical reason for studying underactuated vehicles is that underactuation provides a backup control technique for a fully actuated system. If a fully actuated system is damaged and we have an underactuated controller available, then we may be able to recover gracefully from the failure. The underactuated controller may be able to salvage a system that would otherwise be uncontrollable. This reason for designing controllers would be especially useful for aircraft or spacecraft, where actuator failures can be catastrophic to the vehicle or its mission.

The key steps in this design process were developed by Godhavn $[1,2]$ and we will closely follow his problem formulation and solution technique, but will use a more convenient notation. Our contribution is significant because we 
show how to generalize Godhavn's approach for different types of forces. This generalization is not trivial and we will show how to systematically exploit the backstepping procedure introduced by Godhavn. It is important to study underactuated systems with generalized forces because they represent a more realistic model of the actual vehicle.

As an alternative controller design approach, Pettersen and Nijmeijer [7] have presented another variation of the backstepping technique for a similar problem. Their method allows the ship to recover from initial errors to track a reference trajectory, but they also have considered only one set of forces for the system.

Having introduced the problem and the related literature, we will now outline the remainder of this paper. In Section 2, we formulate the trajectory tracking problem and describe the underactuated ship model. Section 3 presents a detailed solution to the problem using backstepping techniques. We present two simulation results illustrating the approach in Section 4. The final section summarizes the paper and suggests some additional research directions.

\section{Problem Formulation}

We will use a ship model similar to the one presented by Godhavn [1] and make changes to the forces acting on the system.

We start by defining the configuration variable $\mathbf{q}:=[\theta, x, y]^{\mathrm{T}}$ as a vector containing the orientation and position information for the ship with respect to the inertial frame. The velocity vector $\mathbf{v}:=[r, u, v]^{\mathrm{T}}$ is given with respect to the body frame and contains the angular velocity (yaw rate) and the two translational velocities in the forward (surge) and lateral (sway) directions, respectively. We can relate the inertial velocities $\dot{\mathbf{q}}$ and $\mathbf{v}$ through a rotation matrix $\mathbf{R}(\mathbf{q})$ to write the kinematic equations for the ship as follows:

$$
\dot{\mathbf{q}}=\left[\begin{array}{c}
\dot{\theta} \\
\dot{x} \\
\dot{y}
\end{array}\right]=\left[\begin{array}{ccc}
1 & 0 & 0 \\
0 & \cos \theta & -\sin \theta \\
0 & \sin \theta & \cos \theta
\end{array}\right]\left[\begin{array}{c}
r \\
u \\
v
\end{array}\right]=\mathbf{R}(\mathbf{q}) \mathbf{v}
$$

The dynamic equations of motion for the underactuated ship can be written compactly as

$$
\mathbf{M}(\mathbf{q}) \ddot{\mathbf{q}}+\mathbf{C}(\mathbf{q}, \dot{\mathbf{q}}) \dot{\mathbf{q}}=\mathbf{F}(\mathbf{q}),
$$

where $\ddot{\mathbf{q}}$ is the acceleration vector, $\mathbf{M}(\mathbf{q})$ is the inertia matrix, $\mathbf{C}(\mathbf{q}, \dot{\mathbf{q}})$ represents the Coriolis matrix and includes damping terms, and $\mathbf{F}(\mathbf{q})$ represents the forces acting on the vessel. The inertia matrix is invertible, and hence we can solve for the acceleration vector $\ddot{\mathbf{q}}$ as

$$
\ddot{\mathbf{q}}=\mathbf{M}^{-1}(\mathbf{q})[\mathbf{F}(\mathbf{q})-\mathbf{C}(\mathbf{q}, \dot{\mathbf{q}}) \dot{\mathbf{q}}] \text {. }
$$

To develop a specific example, we write the forces as $\mathbf{F}=$ $\mathbf{F}_{1} u_{1}+\mathbf{F}_{2} u_{2}$, where $u_{1}$ and $u_{2}$ are the scalar control inputs, and the vector forces are

$$
\mathbf{F}_{1}=\left[\begin{array}{c}
0 \\
\cos (\theta) \\
\sin (\theta)
\end{array}\right], \quad \mathbf{F}_{2}=\left[\begin{array}{c}
-h \\
-\sin (\theta) \\
\cos (\theta)
\end{array}\right]
$$

The forces correspond to two forces acting at the rear of the ship a distance $h$ from the center of mass. One force is directed along the centerline of the ship and acts as a forward acceleration input from the propeller thrust. The second force is perpendicular to the first and produces both an angular acceleration and a small lateral acceleration for the ship. As mentioned earlier, the lateral acceleration produced by the second force makes the system nonminimum phase when we select $x$ and $y$ as the outputs to track. The model studied by Godhavn uses forces that produce only forward acceleration and torque, so it is minimum phase. Figure 1 illustrates the forces and the other important variables in the model.

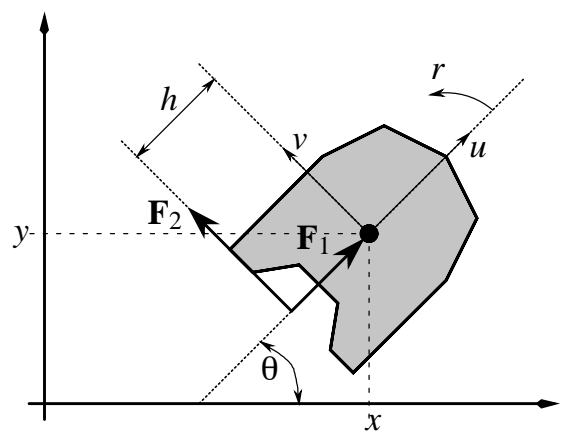

Figure 1: Alignment of forces $\mathbf{F}_{1}$ and $\mathbf{F}_{2}$ with respect to the ship and the inertial reference frame.

We want the underactuated ship to track a given desired trajectory, denoted as $\mathbf{q}_{d}$. We will assume that the desired trajectory is twice continuously differentiable or that it can be closely approximated by a sufficiently smooth trajectory. Godhavn addresses additional aspects of the motion planning process, including generating optimal paths using splines, planning the speed of the vehicle and accounting for actuator saturation limits. We will not address these portions of the problem in this paper.

\section{Backstepping Control Law}

The backstepping analysis for this problem uses vectorial backstepping to reduce the notation. See [5] as a general reference for integrator backstepping. We define the tracking error variable $\mathbf{z}_{1}$ that we would like to drive to zero as the difference between the actual and desired configuration vectors

$$
\mathbf{z}_{1}=\mathbf{q}-\mathbf{q}_{d}
$$

and we take the time derivative of $\mathbf{z}_{1}$ to get

$$
\dot{\mathbf{z}}_{1}=\dot{\mathbf{q}}-\dot{\mathbf{q}}_{d} .
$$


We use this error variable to define a candidate Lyapunov function $V_{1}$ as

$$
V_{1}=\frac{1}{2} \mathbf{z}_{1}^{\mathrm{T}} \mathbf{z}_{1}
$$

We want the time derivative of $V_{1}$ to be negative definite, so we examine $\dot{V}_{1}$ :

$$
\dot{V}_{1}=\mathbf{z}_{1}^{\mathrm{T}} \dot{\mathbf{z}}_{1}=\mathbf{z}_{1}^{\mathrm{T}}\left(\dot{\mathbf{q}}-\dot{\mathbf{q}}_{d}\right) .
$$

Equations (1) and (3) indicate that the inputs $u_{1}$ and $u_{2}$ do not appear in the expression for $\dot{\mathbf{q}}$, but they do appear in $\ddot{\mathbf{q}}$. To pull the control inputs into the design, we need to introduce another error variable and iterate the backstepping procedure. To make $\dot{V}_{1}$ negative definite, it is convenient to introduce the following substitution

$$
\dot{\mathbf{q}}-\dot{\mathbf{q}}_{d}=-c_{1} \mathbf{z}_{1}+\mathbf{z}_{2}
$$

to get

$$
\dot{V}_{1}=-c_{1} \mathbf{z}_{1}^{\mathrm{T}} \mathbf{z}_{1}+\mathbf{z}_{2}^{\mathrm{T}} \mathbf{z}_{1} .
$$

The new error variable is $\mathbf{z}_{2}=\dot{\mathbf{q}}-\dot{\mathbf{q}}_{d}+c_{1} \mathbf{z}_{1}$ as defined by (9). The corresponding Lyapunov function and its time derivative are

$$
\begin{aligned}
V_{2} & =V_{1}+\frac{1}{2} \mathbf{z}_{2}^{\mathrm{T}} \mathbf{z}_{2} \\
\dot{V}_{2} & =\dot{V}_{1}+\mathbf{z}_{2}^{\mathrm{T}} \dot{\mathbf{z}}_{2} \\
& =-c_{1} \mathbf{z}_{1}^{\mathrm{T}} \mathbf{z}_{1}+\mathbf{z}_{2}^{\mathrm{T}}\left(\mathbf{z}_{1}+\dot{\mathbf{z}}_{2}\right) .
\end{aligned}
$$

Computing the time derivative of $\mathbf{z}_{2}$, we get

$$
\dot{\mathbf{z}}_{2}=\ddot{\mathbf{q}}-\ddot{\mathbf{q}}_{d}+c_{1} \dot{\mathbf{z}}_{1} .
$$

Notice that $\ddot{\mathbf{q}}$ appears in (13) and it is a function of the control inputs $u_{1}$ and $u_{2}$. We can use these control inputs to influence the behavior of $\dot{V}_{2}$, but since the system is underactuated, we cannot achieve the type of cancellation that would be used in backstepping for a fully actuated system. If the system were fully actuated, we would rewrite $\dot{V}_{2}$ as

$$
\dot{V}_{2}=-c_{1} \mathbf{z}_{1}^{\mathrm{T}} \mathbf{z}_{1}-c_{2} \mathbf{z}_{2}^{\mathrm{T}} \mathbf{z}_{2}+\mathbf{z}_{2}^{\mathrm{T}}\left(\mathbf{z}_{1}+\dot{\mathbf{z}}_{2}+c_{2} \mathbf{z}_{2}\right)
$$

by adding and subtracting $c_{2} \mathbf{z}_{2}^{\mathrm{T}} \mathbf{z}_{2}$. We would then use the inputs to eliminate the term in parentheses in (14). In our case, however, we have only two inputs and, in general, cannot completely cancel the three dimensional vector in the parentheses.

Godhavn resolves this problem through a series of design choices for the control law. The key step in the approach is to apply the backstepping process to track only two position variables instead of the entire three dimensional configuration. The next step is to select the forces so that the control inputs appear one at a time as we iterate the backstepping procedure $^{1}$. In conjunction with the first step, the second

\footnotetext{
${ }^{1}$ Godhavn actually fixed the forces at the start of the problem formulation, but he could have selected other force configurations.
}

step allows us to easily cancel terms to achieve tracking for the two position variables. The third and final step is to select desired trajectories that yield stable zero dynamics for the orientation variable. With stable zero dynamics and the backstepping controller, the ship will asymptotically track position as well as orientation, provided we start with suitable initial conditions.

Godhavn demonstrates his approach for a system with a pure acceleration and a pure torque as the force inputs. We explain how to generalize the technique for the case when the input forces and torques are coupled. We consider the force configuration as a fixed part of the system that we must design around. To find a solution, we need to select two outputs that cause the control inputs to appear one at a time during the backstepping procedure. To understand how we developed the outputs for this approach, we will re-derive the backstepping equations with one modification. The change is that the output we will track is now two dimensional instead of being three dimensional. Accordingly, we have a desired trajectory that is also two dimensional. The output and the desired trajectory are denoted by $\mathbf{h}(\mathbf{q})$ and $\mathbf{h}_{d}\left(\mathbf{q}_{d}\right)$, respectively.

We start the backstepping procedure with error variable $\mathbf{z}_{1}$ :

$$
\mathbf{z}_{1}=\mathbf{h}-\mathbf{h}_{d}
$$

and define a Lyapunov function as

$$
V_{1}=\frac{1}{2} \mathbf{z}_{1}^{\mathrm{T}} \mathbf{z}_{1} .
$$

The backstepping procedure is very similar to the one described in detail above, so we will present the equations below with only brief comments. First, take the time derivative of $V_{1}$ :

$$
\dot{V}_{1}=\mathbf{z}_{1}^{\mathrm{T}} \dot{\mathbf{z}}_{1}=\mathbf{z}_{1}^{\mathrm{T}}\left(\dot{\mathbf{h}}-\dot{\mathbf{h}}_{d}\right) .
$$

Set $\mathbf{z}_{2}-c_{1} \mathbf{z}_{1}=\dot{\mathbf{h}}-\dot{\mathbf{h}}_{d}$ to get

$$
\dot{V}_{1}=-c_{1} \mathbf{z}_{1}^{\mathrm{T}} \mathbf{z}_{1}+\mathbf{z}_{2}^{\mathrm{T}} \mathbf{z}_{1} .
$$

Choose

$$
V_{2}=V_{1}+\frac{1}{2} \mathbf{z}_{2}^{\mathrm{T}} \mathbf{z}_{2}
$$

which gives

$$
\begin{aligned}
\dot{V}_{2} & =-c_{1} \mathbf{z}_{1}^{\mathrm{T}} \mathbf{z}_{1}+\mathbf{z}_{2}^{\mathrm{T}}\left[\mathbf{z}_{1}+\dot{\mathbf{z}}_{2}\right] \\
& =-c_{1} \mathbf{z}_{1}^{\mathrm{T}} \mathbf{z}_{1}+\mathbf{z}_{2}^{\mathrm{T}}\left[\mathbf{z}_{1}+\ddot{\mathbf{h}}-\ddot{\mathbf{h}}_{d}+c_{1}\left(\dot{\mathbf{h}}-\dot{\mathbf{h}}_{d}\right)\right] .
\end{aligned}
$$

The output $\mathbf{h}$ is a function of $\mathbf{q}$ and the inputs appear in $\ddot{\mathbf{q}}$, so they also appear in the second derivative of $\mathbf{h}$, as shown below. Consider

$$
\begin{aligned}
& \dot{\mathbf{h}}=\frac{d \mathbf{h}(\mathbf{q})}{d t}=\frac{\partial \mathbf{h}}{\partial \theta} \dot{\theta}+\frac{\partial \mathbf{h}}{\partial x} \dot{x}+\frac{\partial \mathbf{h}}{\partial y} \dot{y}=d \mathbf{h} \cdot \dot{\mathbf{q}} \\
& \ddot{\mathbf{h}}=\frac{d^{2} \mathbf{h}(\mathbf{q})}{d t^{2}}=d \mathbf{h} \cdot \ddot{\mathbf{q}}+\frac{D^{2} \mathbf{h}}{\partial \mathbf{q} \partial \mathbf{q}}(\dot{\mathbf{q}}, \dot{\mathbf{q}})
\end{aligned}
$$


where $d \mathbf{h}$ is the differential of $\mathbf{h}$ with respect to $\mathbf{q}$ and $\frac{D^{2} \mathbf{h}}{\partial \mathbf{q} \partial \mathbf{q}}$ is the matrix of second partial derivatives of $\mathbf{h}$.

Using (3) and $\mathbf{F}=\mathbf{F}_{1} u_{1}+\mathbf{F}_{2} u_{2}$, we see that

$$
\ddot{\mathbf{q}}=\mathbf{M}^{-1} \mathbf{F}_{1} u_{1}+\mathbf{M}^{-1} \mathbf{F}_{2} u_{2}-\mathbf{M}^{-1} \mathbf{C} \dot{\mathbf{q}} .
$$

To simplify notation, let $\mathbf{Y}_{1}:=\mathbf{M}^{-1} \mathbf{F}_{1}$, and $\mathbf{Y}_{2}:=\mathbf{M}^{-1} \mathbf{F}_{2}$. The expression for $\ddot{\mathbf{h}}$ has the term $d \mathbf{h} \cdot \ddot{\mathbf{q}}$. Using the above simplifications, to have control input $u_{1}$ appear in $\ddot{\mathbf{h}}$ and input $u_{2}$ not appear, we require

$$
d \mathbf{h} \cdot \mathbf{Y}_{1} \neq 0 \quad \text { and } \quad d \mathbf{h} \cdot \mathbf{Y}_{2}=0
$$

for the output $\mathbf{h}$. Similarly, for control input $u_{2}$ to appear in $\ddot{\mathbf{h}}$ and input $u_{1}$ not to appear, we require

$$
d \mathbf{h} \cdot \mathbf{Y}_{1}=0 \quad \text { and } \quad d \mathbf{h} \cdot \mathbf{Y}_{2} \neq 0 .
$$

To find outputs that satisfy these conditions, we first compute $\mathbf{Y}_{1}$ and $\mathbf{Y}_{2}$ as

$$
\mathbf{Y}_{1}=\left[\begin{array}{c}
0 \\
\frac{\cos (\theta)}{m_{x}} \\
\frac{\sin (\theta)}{m_{x}}
\end{array}\right], \quad \mathbf{Y}_{2}=\left[\begin{array}{c}
-\frac{h}{J} \\
-\frac{\sin (\theta)}{m_{y}} \\
\frac{\cos (\theta)}{m_{y}}
\end{array}\right]
$$

where $m_{x}$ and $m_{y}$ are the mass terms, including added mass, for the ship and $J$ is the moment of inertia for the ship. To make $d \mathbf{h} \cdot \mathbf{Y}_{1}=0$, consider the nullspace of $\mathbf{Y}_{1}$ :

$$
\mathcal{N}\left(\mathbf{Y}_{1}\right)=\operatorname{span}\left\{\left[\begin{array}{l}
1 \\
0 \\
0
\end{array}\right],\left[\begin{array}{c}
0 \\
-\sin (\theta) \\
\cos (\theta)
\end{array}\right]\right\} .
$$

We want to pick outputs such that their differentials are in the nullspace of $\mathbf{Y}_{1}$. The existence of such functions and their computation are discussed in Remark 2 below. Two such outputs that satisfy $d \mathbf{h} \cdot \mathbf{Y}_{1}=0$ are

$$
h_{11}=\theta, \quad h_{12}=-x \sin (\theta)+y \cos (\theta) .
$$

A similar analysis for $\mathbf{Y}_{2}$ shows

$$
\mathcal{N}\left(\mathbf{Y}_{2}\right)=\operatorname{span}\left\{\left[\begin{array}{c}
\frac{J}{h m_{y}} \cos (\theta) \\
0 \\
1
\end{array}\right],\left[\begin{array}{c}
-\frac{J}{h m_{y}} \sin (\theta) \\
1 \\
0
\end{array}\right]\right\}
$$

which leads to two outputs satisfying $d \mathbf{h} \cdot \mathbf{Y}_{2}=0$ as

$$
h_{21}=x+\frac{J}{h m_{y}} \cos (\theta), \quad h_{22}=y+\frac{J}{h m_{y}} \sin (\theta) .
$$

We now have two pairs of outputs that will allow $u_{1}$ and $u_{2}$ to appear one at a time in the backstepping procedure. We can then rewrite (15) as:

$$
\dot{V}_{2}=-c_{1} \mathbf{z}_{1}^{\mathrm{T}} \mathbf{z}_{1}-c_{2} \mathbf{z}_{2}^{\mathrm{T}} \mathbf{z}_{2}+\mathbf{z}_{2}^{\mathrm{T}}\left[\mathbf{z}_{1}+\dot{\mathbf{z}}_{2}+c_{2} \mathbf{z}_{2}\right] .
$$

We recall that $\dot{\mathbf{z}}_{2}$ contains $\ddot{\mathbf{h}}$. Suppose we select $\mathbf{h}_{1}=$ $\left[h_{11}, h_{12}\right]^{\mathrm{T}}$ as our output to track and define $\mathbf{h}_{1 d}$ as

$$
\mathbf{h}_{1 d}:=\left[\begin{array}{c}
\theta_{d} \\
-x_{d} \sin \left(\theta_{d}\right)+y_{d} \cos \left(\theta_{d}\right)
\end{array}\right]
$$

which is $\mathbf{h}_{1}$ with $[\theta, x, y]^{\mathrm{T}}$ replaced by $\left[\theta_{d}, x_{d}, y_{d}\right]^{\mathrm{T}}$. By construction, we have $d h_{11} \cdot \mathbf{Y}_{1}=d h_{12} \cdot \mathbf{Y}_{1}=0$, so $u_{1}$ does not appear in $\dot{V}_{2}$. We can now use $u_{2}$ to cancel one element in the two dimensional vector $\left[\mathbf{z}_{1}+\dot{\mathbf{z}}_{2}+c_{2} \mathbf{z}_{2}\right]$. Following Godhavn [1], we will choose to cancel the first component and denote the remaining element with $a$. We now define a third error variable, $\mathbf{z}_{3} \in \mathbb{R}$ as

$$
\mathbf{z}_{3}=\frac{a}{\lambda}
$$

where $\lambda$ is a positive design parameter introduced by Godhavn [1] that influences the exponential rate of decay for the error variables. We let

$$
V_{3}=V_{2}+\frac{1}{2} \mathbf{z}_{3}^{2}
$$

and calculate

$$
\dot{V}_{3}=-c_{1} \mathbf{z}_{1}^{\mathrm{T}} \mathbf{z}_{1}-c_{2} \mathbf{z}_{2}^{\mathrm{T}} \mathbf{z}_{2}+\mathbf{z}_{2}^{\mathrm{T}}\left[\begin{array}{l}
0 \\
a
\end{array}\right]+\mathbf{z}_{3} \dot{\mathbf{z}}_{3},
$$

where we have applied $u_{2}$ to zero the first element in $\left[\mathbf{z}_{1}+\right.$ $\left.\dot{\mathbf{z}}_{2}+c_{2} \mathbf{z}_{2}\right]$. We note that $\dot{\mathbf{z}}_{3}=\dot{a} / \lambda$ and that $\dot{a}$ will contain both $u_{1}$ and $u_{2}$, so we can pick $u_{1}$ to cancel one other term. We use $u_{1}$ to set

$$
\dot{\mathbf{z}}_{3}=-c_{3} \mathbf{z}_{3}-\mathbf{z}_{2}^{\mathrm{T}}\left[\begin{array}{l}
0 \\
\lambda
\end{array}\right],
$$

so that

$$
\mathbf{z}_{3} \dot{\mathbf{z}}_{3}=-c_{3} \mathbf{z}_{3}^{2}-\mathbf{z}_{2}^{\mathrm{T}}\left[\begin{array}{c}
0 \\
\lambda \mathbf{z}_{3}
\end{array}\right]=-c_{3} \mathbf{z}_{3}^{2}-\mathbf{z}_{2}^{\mathrm{T}}\left[\begin{array}{l}
0 \\
a
\end{array}\right],
$$

and

$$
\dot{V}_{3}=-c_{1} \mathbf{z}_{1}^{\mathrm{T}} \mathbf{z}_{1}-c_{2} \mathbf{z}_{2}^{\mathrm{T}} \mathbf{z}_{2}-c_{3} \mathbf{z}_{3}^{2} .
$$

With the above choices for $u_{1}$ and $u_{2}$, our controller will drive the $\mathbf{z}_{i}$ error variables to the origin. In fact, if we let $\mathbf{b}=[0, \lambda]^{\mathrm{T}}$, we can write the closed-loop equations for the error dynamics as

$$
\left[\begin{array}{l}
\dot{\mathbf{z}}_{1} \\
\dot{\mathbf{z}}_{2} \\
\dot{\mathbf{z}}_{3}
\end{array}\right]=\left[\begin{array}{ccc}
-c_{1} \mathbf{I} & \mathbf{I} & 0 \\
-\mathbf{I} & -c_{2} \mathbf{I} & \mathbf{b} \\
0 & -\mathbf{b}^{\mathrm{T}} & -c_{3}
\end{array}\right]\left[\begin{array}{l}
\mathbf{z}_{1} \\
\mathbf{z}_{2} \\
\mathbf{z}_{3}
\end{array}\right] .
$$

Using standard arguments, we can show that the $\mathbf{z}$-system is exponentially stable and the tracking error $\mathbf{h}_{1}-\mathbf{h}_{1 d} \rightarrow 0$. Several remarks about this approach are in order.

Remark 1. We could perform a similar analysis using outputs $h_{21}$ and $h_{22}$, which would swap the order in which $u_{1}$ and $u_{2}$ appear in the procedure.

Remark 2. For our system, given any force $\mathbf{F}$ and an inertia matrix $\mathbf{M}$, we can compute $\mathbf{Y}=\mathbf{M}^{-1} \mathbf{F}$, because the inertia matrix is always invertible. If we consider $\mathbf{Y}$ as a vector field in $\mathbb{R}^{3}$, then it is nonsingular and involutive. The Frobenius theorem [4, p. 23] implies that we can always 
find two functions $h_{a}(\mathbf{q})$ and $h_{b}(\mathbf{q})$ such that $d h_{a} \cdot \mathbf{Y}=0$ and $d h_{b} \cdot \mathbf{Y}=0$. We can use these two functions to form $\mathbf{h}=\left[h_{a}, h_{b}\right]^{\mathrm{T}}$ and then apply the backstepping procedure. Hence, we can always find outputs that allow us to use this solution technique for a mechanical system with three degrees of freedom and two inputs, regardless of the structure of the forces applied to the vehicle. The outputs we constructed yield a system with relative degree $(2,3)$ [4]. The Frobenius theorem would also be instrumental in extending these results to higher order systems.

Remark 3. The overall design approach is flexible enough to handle a variety of cases. The flexibility comes from by being able to choose the outputs to track, swapping the order of appearance of $u_{1}$ and $u_{2}$ in the design, or by modifying the desired trajectory. These design choices allow us to efficiently prevent several potential problems. For example, one possible problem we can dismiss is that driving the $\mathbf{z}_{i}$ variables to zero does not imply that $\theta \rightarrow \theta_{d}, x \rightarrow x_{d}$ and $y \rightarrow y_{d}$. Since the $\mathbf{z}_{i}$ error variables depend on the outputs, and the outputs may contain trigonometric functions, it is possible to find combinations of configuration variables that drive the error variables to zero but do not track the desired trajectory. Another key requirement in this approach is that the coefficients on $u_{1}$ and $u_{2}$ must be nonzero when we are solving for them in the appropriate equations. Our analysis and simulations show that this is not always the case, which is a second potential problem that can be easily corrected in the design. A third potential problem that can also be addressed is that the control inputs can grow very large under certain configurations. This type of problem usually can be corrected by modifying the desired trajectory.

Carefully selecting the outputs and the desired trajectories allows us to apply Godhavn's basic backstepping technique to solve the underactuated tracking problem for a ship with generalized forces. We have remarked that the approach will work for any set of forces, including those that introduce a nonminimum phase property for the vehicle. Our presentation highlights a systematic procedure to compute the tracking outputs that allow us to solve the problem. We have noted a few difficulties that may arise, but the design process is flexible enough to overcome these minor issues. We will now present the simulation results that highlight some of the advantages and disadvantages of the approach.

\section{Simulation Results}

We simulated the backstepping design approach for a simple underactuated ship model where the angular and lateral acceleration inputs were coupled. To perform trajectory tracking, we selected output $\mathbf{h}_{2}$ as described in Section 3. The desired trajectories include straight lines and circles and we assume the ship is always in motion. Godhavn [1] shows how to use line segments and arcs of circles to construct splines representing efficient trajectories.
Figure 2 shows the vehicle accurately tracking a linear trajectory with the proper orientation after starting with an initial orientation error. The vehicle starts on the desired trajectory, but it is pointing in the $-x$ direction. The vehicle initially moves backwards (in the $+x$ direction) and corrects the orientation before tracking the desired path. This simulation highlights the fact that the approach has the ability to correct for poor initial orientations. For certain initial conditions, the method used by Godhavn [1] allows the ship to track the correct path while oriented backwards.

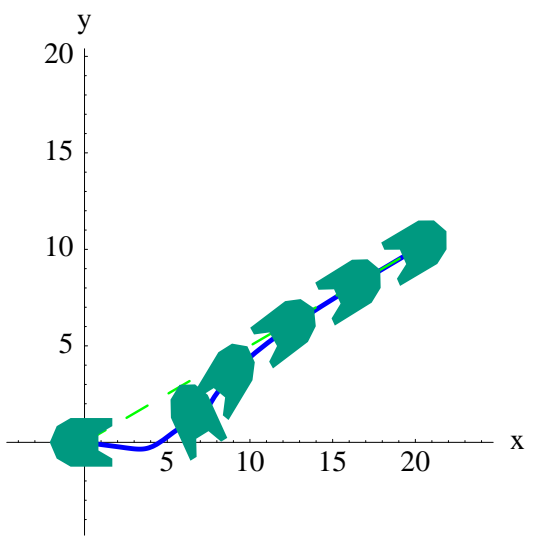

Figure 2: Trajectory tracking with an underactuated vehicle. The vehicle moves backwards and rotates to track the desired trajectory with the correct orientation.

The next simulation illustrates one of the apparent drawbacks of the approach and gives us a chance to explain how a simple adjustment to the design process addresses this issue. Figure 3 displays an attempt to track a circular path. The vehicle starts outside the desired path and moves to a trajectory on a concentric smaller circle. The vehicle is not oriented along its direction of motion and the nose always points toward the desired path. Changing the initial conditions did not affect the steady-state motion of the vehicle in this example. This stable motion arises because of the outputs we selected and the shape of the desired trajectory. As mentioned above, it is possible that the $\mathbf{z}_{i}$ error variables could be driven to zero, but the values of $\theta, x$ and $y$ may not match those of $\theta_{d}, x_{d}$ and $y_{d}$.

To better understand this type of behavior, we will outline the procedure for describing the motion. We will not present the details for this example because the expressions are complicated and do not aid in understanding the results. For a given output $\mathbf{h}(\mathbf{q})$, construct $\mathbf{h}_{d}\left(\mathbf{q}_{d}\right)$, by substituting the desired configuration variables for the actual configuration variables. Form the following three equations

$$
\begin{aligned}
\mathbf{h}-\mathbf{h}_{d} & =0 \\
\dot{\mathbf{h}}-\dot{\mathbf{h}}_{d} & =0 \\
a & =0
\end{aligned}
$$

where $a$ comes from (18). Since $\mathbf{h}$ and $\mathbf{h}_{d}$ are two dimensional vectors, expressions (20) through (22) represent five 
scalar equations. We are interested in solving for the three configuration variables and their derivatives, or a total of six terms. Solving the five scalar equations (20)-(22) will explain the motion of vehicle as it attempts to track the desired trajectory. There will be one additional degree of freedom in the problem, which will determine the tracking performance. (By a slight abuse of terminology, this additional degree of freedom can be characterized as the zero dynamics of the system [4, p. 164].) The solution of (20) through (22) may indicate perfect tracking, as shown in Figure 2, or it could allow for the tracking behavior shown in Figure 3.

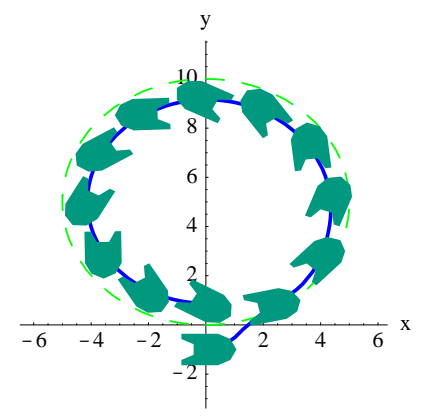

Figure 3: While tracking a circular path, the vehicle maneuvers to a steady configuration inside the desired path with the nose of the vehicle pointed toward the desired path.

The predictable type of motion generated when the vehicle attempts to track a circle may still be useful in designing trajectories for our vehicle. Since the resulting path is also a circle, to get the ship to track a circle, we just need to specify a desired trajectory with a larger radius than that of the path we want the ship to track. The correction factor will depend on the mass and damping properties of the ship, the speed, and the radius of curvature of the intended path. As indicated earlier, we can compensate for imperfect tracking by carefully selecting the desired trajectory for the system.

The simulations illustrate the strengths and weaknesses of the backstepping design approach applied to an underactuated system with generalized forces. By selecting desired trajectories composed of line segments and appropriately scaled arcs of circles, we can use the approach to efficiently control the ship's motion between two configurations.

\section{Conclusions and Future Work}

This paper describes a generalization to an existing backstepping controller design and solves the tracking problem for a nonlinear underactuated system. The approach presents a systematic method for selecting outputs based on a geometrical analysis of the forces acting on the system. This contribution provides an extension to the current results, but is limited in the types of trajectories it can track. The simulations illustrate the positive aspects of the approach, as well as some of the potential drawbacks. We explain how to compensate for the drawbacks by carefully choosing the tracking outputs and the desired trajectories.

There are several areas of research related to this problem that deserve attention. The approach could be further generalized by considering the output feedback case where the states are estimated from measurements. In addition, to make the model more realistic, the equations of motion and measurement equations should include disturbances, which will substantially change the nature of the problem. Another research option is to account for the underactuated nature of the vehicle when generating the desired trajectories to improve the tracking performance.

\section{Acknowledgments}

This research was partly support by the U.S. Department of Energy Grant DOE-DEFG-02-97ER13939.

\section{References}

[1] J-M. Godhavn. Nonlinear tracking of underactuated surface vessels. In Proceedings of the 35th IEEE Conference on Decision and Control, volume 1, pages 975-980. IEEE, December 1996.

[2] J.-M. Godhavn. Topics in Nonlinear Motion Control: Nonholonomic, Underactuated and Hybrid Systems. PhD thesis, Norwegian University of Science and Technology, N-7034 Trondheim, Norway, 1997.

[3] J. Hauser, S. Sastry, and G. Meyer. Nonlinear control design for slightly non-minimum phase systems: application to V/STOL aircraft. Automatica, 28(4):665-679, July 1992.

[4] A. Isidori. Nonlinear Control Systems. Springer, Great Britain, Third edition, 1995.

[5] M. Krstić, I. Kanellakopoulos, and P. Kokotović. Nonlinear and Adaptive Control Design. John Wiley \& Sons, Inc., New York, 1995.

[6] N. E. Leonard. Compensating for actuator failures: Dynamics and control of underactuated underwater vehicles. In 9th International Symposium on Unmanned Untethered Submersible Technology, pages 168-177, September 1995.

[7] K. Y. Pettersen and H. Nijmeijer. State tracking control of an underactuated surface vessel. In Proceedings of the 37th IEEE Conference on Decision and Control, pages 4561-4566, Tampa, Florida, December 1998. IEEE.

[8] M. Reyhanoglu. Exponential stabilization of an underactuated autonomous surface vessel. Automatica, 33(12):2249-2254, December 1997.

[9] M. Reyhanoglu, A. van der Schaft, N. H. McClamroch, and I. Kolmanovsky. Dynamics and control of a class of underactuated mechanical systems. IEEE Transactions on Automatic Control, 44(9):1663-1671, September 1999. 